\title{
Dynamic Changes in the Proximal Gut Neural Crest Stem Cell Population Are Associated with Successful Development of the Distal Enteric Nervous System in Rats
}

\author{
YU-HWAI TSAI AND CHERYL E. GARIEPY \\ Department of Pediatrics and Communicable Diseases, University of Michigan, Ann Arbor, MI 48109
}

\begin{abstract}
Loss of signaling through the endothelin-B receptor $\left(\mathrm{ET}_{\mathrm{B}}\right)$ leads to failure of vagal neural crest $(\mathrm{NC})$ cell colonization of the developing gut and causes congenital distal intestinal aganglionosis [Hirschsprung disease (HSCR)] in humans and other mammals. Several studies suggest that cell-cell interactions and the number of NC cells behind the wavefront may play an important role in successful gut colonization. We compared the number and progression of enteric nervous system stem cells in the wild-type (WT) and HSCR rat gut using whole-mount immunohistochemistry for $\mathrm{p} 75$, culture and isolation of NC stem cells (NCSCs) by flow cytometry. Isolation and culture demonstrates that NCSCs enter the WT cecum between embryonic day (E) 13.5 and E14.5, and the number of NCSC in the colon significantly increases after E15.5. These findings are consistent with the caudal progression of the NC-cell wavefront by wholemount staining. During the period of WT colonic colonization of the proximal colon, we found significant differences in the small bowel NCSC pool between WT and HSCR rats. Whereas the

proximal gut NCSC pool in WT rats is increasing behind the colonization wavefront, no such change occurs in the proximal NCSC pool in HSCR rats. Dynamic changes in the NCSC pool occur behind the NC colonization wavefront in the gut of WT rats. The absence of these changes in the HSCR rat may contribute to distal aganglionosis. (Pediatr Res 58: 636-643, 2005)
E, embryonic day
ENS, enteric nervous system
$\mathbf{E T}_{\mathbf{B}}$, endothelin-B receptor
FACS, fluorescence-activated cell sorting
HSCR, Hirschsprung disease
NC, neural crest
NCSC, neural crest stem cells
WT, wild-type
$\mathbf{E T}_{\mathbf{3}}$, endothelin 3

Abbreviations
\end{abstract}

Hirschsprung disease (HSCR) is a congenital abnormality of the enteric nervous system (ENS) and is the most common cause of congenital intestinal obstruction. HSCR is characterized by distal intestinal aganglionosis, and current treatment involves resection of the aganglionic segment of bowel. HSCR affects 1 in every 5000 children born worldwide and is associated with a variety of other congenital anomalies and syndromes. Persistent problems with enterocolitis, diarrhea, constipation, or encopresis are common after resection. Rarely, the length of aganglionic bowel is so great that resection leaves insufficient bowel for nutrient absorption. Morbidity and mor-

Received November 8, 2004; accepted March 16, 2005.

Correspondence: Cheryl E. Gariepy, M.D., Department of Pediatrics and Communicable Diseases, University of Michigan, Medical Science Research Building I, A520, Ann Arbor, MI 48109-0656; e-mail: CGariepy@med.umich.edu.

Supported by the Wayne Jones Family Fund, The Michigan Gut Peptide Research Center, The Mott Children's Hospital Research Fund

DOI: 10.1203/01.PDR.0000180552.12737.9F tality from this disease remain high, even in developed countries (1).

All of the neurons and glia of the ENS are derived from the neural crest (NC). The majority of enteric ganglia are derived from vagal $\mathrm{NC}$ cells that colonize the developing gut in a cranial-to-caudal progression. HSCR occurs when vagal NC cells fail to complete colonization of the distal gut. Several animal models of HSCR exist, and mutations in eight distinct genes are associated with this developmental abnormality in mammals. At least $10 \%$ of human HSCR is cause by defective signaling through endothelin-B receptor $\left(\mathrm{ET}_{\mathrm{B}}\right)(2)$. The $\mathrm{ET}_{\mathrm{B}^{-}}$ null rat is a naturally occurring model of total colonic HSCR $(3,4)$. Postnatally, at least $90 \%$ of animals have no ganglion cells beyond the ileocecal junction (3).

Several groups have recently developed techniques to isolate and culture multipotent ENS precursors $(5,6)$. Bixby et al. (6) developed a technique to prospectively identify and isolate enteric NC stem cells (NCSCs) by fluorescence-activated cell sorting (FACS) for high expression of p75, the neurotrophin 
receptor, and $\alpha_{4}$ integrin. These cells are self-renewing, comprise only a few percent of fetal gut cells (6), can be identified in the proximal gut of the HSCR rat postnatally, and demonstrate neuronal development after injection into the embryonic distal gut of HSCR rats in organ culture (7). Although it is established that wild-type (WT) gut NCSCs express $\mathrm{ET}_{\mathrm{B}}$ (8) and the mesenchyme of the developing gut expresses its ligand, endothelin-3, the role of $\mathrm{ET}_{\mathrm{B}}$ in ENS development at the cellular level remains controversial. An absence of $\mathrm{ET}_{\mathrm{B}}$ signaling may lead to a primary defect in the ability of NCderived cells to migrate $(7,9)$ or to a secondary reduction in the ENS progenitor pool through premature differentiation or proliferation failure (10-12).

The colonization behavior of NC-derived cells is influenced by intrinsic factors, molecules expressed by the gut mesenchyme, and interactions between NC cells. The nature and necessity of NC cell-cell interactions in intestinal colonization is just beginning to be examined. Recently, Young et al. (13) demonstrated that when a small population of the most distal hindgut NC-derived cells is isolated from the population behind them, they migrated more slowly. These and similar results support the idea that population pressure behind the wavefront may stimulate distal migration of ENS precursors $(14,15)$. We undertook a series of experiments to examine the localization and dynamics of the gut NCSC population in WT and HSCR rats.

\section{METHODS}

Animals. Protocols were conducted in accordance with National Institutes of Health guidelines and approved and monitored by the University of Michigan University Committee on the Use and Care of Animals. The $\mathrm{ET}_{\mathrm{B}}$-null mutation, $E T_{B}{ }^{s l}$ is maintained on an inbred Wistar-Kyoto genetic background. The $E T_{B}{ }^{s l}$ mutation is autosomal recessive. $E T_{B}{ }^{s l} / s l$ rats develop intestinal obstruction in the neonatal period, do not live beyond $36 \mathrm{~d}$, and are referred to as HSCR rats throughout this report. $\mathrm{ET}_{\mathrm{B}}$ heterozygous rats are indistinguishable from WT rats visually, in lifespan, and in fertility. $\mathrm{ET}_{\mathrm{B}}$ genotype was determined by PCR analysis of a tail biopsy specimen or, in the case of embryos, from unused portions of the embryo as previously described (16). Embryos were obtained by timed heterozygous mating and killing of the pregnant female rat. Embryos were immediately dissected, with collection of the entire intestine from the esophagogastric junction to the rectum.

Whole-mount immunohistochemistry. Whole embryonic intestines were fixed in $4 \%$ formaldehyde for $3 \mathrm{~h}$ at room temperature or overnight at $4{ }^{\circ} \mathrm{C}$. After washing with PBS, guts were treated with collagenase $(1 \mathrm{mg} / \mathrm{mL})$ for 30 min. The intestines then were incubated for $1 \mathrm{~h}$ with $0.5 \% \mathrm{BSA}, 1 \% \mathrm{DMSO}$, $0.2 \%$ triton-X100, and 5\% goat serum in PBS. Rabbit anti-mouse p75 (Chemicon AB1554, Temecula, CA) was added directly to the blocking buffer and incubated at $4^{\circ} \mathrm{C}$ overnight. After washing with blocking buffer (without goat serum), the intestines were incubated with the secondary antibody, goat anti-rabbit IgG conjugated with HRP (PI-1000; Vector Lab Inc., Burlingame, $\mathrm{CA})$ for $2 \mathrm{~h}$ at room temperature. They then were washed with acetateimidazole buffer (17.5\% $1 \mathrm{M}$ sodium acetate and 5\% $0.2 \mathrm{M}$ imidazole) followed by Ni-DAB buffer (12.4\% $1 \mathrm{M}$ sodium acetate, $5 \% 0.2 \mathrm{M}$ imidazole, $10 \% 1 \mathrm{M}$ nickel sulfate, $3 \% 10 \mathrm{mg} / \mathrm{mL}$ diaminobenzidine, and $0.3 \%$ of $1 \%$ $\mathrm{H}_{2} \mathrm{O}_{2}$ ).

The stained intestines were examined and measured under a dissecting microscope. They were marked into three small intestinal segments of equal length, two cecal segments of equal length, and four large intestinal segments of equal length. The last division of the large bowel is also referred to as rectum. Each section then was individually examined and scored $(0-5)$ for the density of staining in a genotype-blinded manner.

Flow cytometry and cell sorting. Freshly isolated whole intestines were dissected into cold $\mathrm{Ca}^{2+}$ - and $\mathrm{Mg}^{2+}$-free HBSS and divided at the ileocecal junction and just distal to the cecum, forming three segments that are designated in the figures as proximal, cecal, and distal. Intestinal segments were dissociated by incubating for $2 \mathrm{~min}$ at $37^{\circ} \mathrm{C}$ in $0.5 \mathrm{mg} / \mathrm{mL}$ deoxyribonuclease I (Sigma Chemical Co.) in $\mathrm{Ca}^{2+}$ - and $\mathrm{Mg}^{2+}$-free HBSS. Cells were stained with antibodies against p75 (192I clone) and $\alpha_{4}$ (Becton-Dickinson, San Jose, $\mathrm{CA}$; Mr $\alpha 4-1$ clone, directly conjugated to phycoerythrin). After washing, cells were resuspended in staining medium that contained $2 \mu \mathrm{g} / \mathrm{mL} 7$-aminoactinomycin D (7-AAD; Molecular Probes, Eugene, OR). Cell sorting and analysis were performed on a FACSVANTAGE dual-laser flow-cytometer (BectonDickinson). Dead cells were eliminated from sorts and analysis as 7-AAD ${ }^{+}$.

Sorts were performed using two methods for setting the gates. To compare the number of cells with defined levels of expression of p75 and $\alpha_{4}$ between the two genotypes, we set the gates to collect the top $1 \%$ of cells that expressed these markers in the proximal WT gut sections of the same age, stained and sorted on the same day, and all suspended cells were counted. These results are presented in Figs. $3 A, 4 A, 5 A$. To address the possibility that $\mathrm{ET}_{\mathrm{B}}$ deficiency results in a lower level of expression of p75 or $\alpha_{4}$ without truly affecting the number of multipotent NCSCs, we performed other sorts with the gate set to collect the top $1 \%$ of cells that expressed p75 and $\alpha_{4}$ in the proximal section of that embryo, whether WT or HSCR. This gate resulted in the selection of more cells in HSCR embryos compared with the previously described gate.

Culture of NCSCs. Cells were cultured as previously described (6). Plates were sequentially coated with $150 \mu \mathrm{g} / \mathrm{mL}$ poly-D-lysine (Biomedical Technologies, Stoughton, MA) and $0.15 \mathrm{mg} / \mathrm{mL}$ human fibronectin (Biomedical Technologies) as described (17). Culture plates were kept in sealed plastic bags that contained $6 \% \mathrm{CO}_{2}$ before and after sorting to maintain a physiologic $\mathrm{pH}$ of the culture medium. Cells were cultured on six-well plates at low density $(<30$ clones per well). The culture medium was DMEM-low (GIBCO 11885084 ) with $15 \%$ chick embryo extract, $20 \mathrm{ng} / \mathrm{mL}$ recombinant human basic fibroblast growth factor (R\&D Systems, Minneapolis, MN), $1 \%$ N2 supplement (GIBCO), 2\% B27 supplement (GIBCO), $50 \mu \mathrm{M}$ 2-mercaptoethanol, 110 $\mathrm{nM}$ retinoic acid (Sigma Chemical Co.), penicillin/streptomycin (Cambrex BioScience Rockland, Rockland, ME), and 20 ng/mL IGF-1 (R\&D Systems). After $6 \mathrm{~d}$, cells were switched to "differentiation" medium, which contains $1 \%$ chick embryo extract, $10 \mathrm{ng} / \mathrm{mL}$ basic fibroblast growth factor, and no IGF-1. All cultures were maintained in gas-tight chambers (Billups-Rothenberg, Del-Mar, CA) that contained decreased oxygen levels, as previously described (18).

Immunohistochemistry of cultured cells. Cultures were fixed in acid ethanol (5\% glacial acetic acid, 95\% ethanol) for $20 \mathrm{~min}$ at $-20^{\circ} \mathrm{C}$, washed, blocked, and triply labeled for Peripherin (Chemicon AB1530) to identify neurons, glial fibrillary acid protein (Sigma Chemical Co. G-3893) to identify glia, and $\alpha$-smooth muscle actin (Sigma Chemical Co. A-2547) to identify myofibroblasts.

Cell-cycle analysis. To examine whether the decreased number of NCSCs in the HSCR stomach and small intestine results from decreased proliferation of these cells in vivo, we performed cell-cycle analysis as previously described $(19,20)$. For this experiment, we examined the top $1 \%$ of cells that expressed p75 and $\alpha_{4}$ in the stomach and proximal four fifths of the small intestine. Dissociated gut cells were suspended in medium that contained $5 \mu \mathrm{g} / \mathrm{mL}$ Hoechst 33342 (Sigma Chemical Co.). Cells were incubated in this solution for $45 \mathrm{~min}$ at $37^{\circ} \mathrm{C}$ with agitation every $5-10 \mathrm{~min}$. Cells were kept on ice at all times while stained for flow cytometry analysis.

Statistics. All results are presented as the mean \pm SEM, and $p<0.05$ is considered significant. Data were analyzed using GraphPad Prism version 3.00 for Windows (GraphPad Software, San Diego, CA). Comparisons between two groups were made using the nonparametric Mann-Whitney test. Three or more groups were compared using ANOVA.

\section{RESULTS}

Localization of NCSCs correlates with vagal NC colonization of the WT rat intestine. Whole-mount immunohistochemistry for p75 identifies NCSCs, neural progenitors, and mature neurons. We used the technique to identify the NCSC colonization wavefront in WT and HSCR rats. The density and the intensity of the staining were determined on a six-point scale by an observer who was blinded to intestinal location and embryo genotype. Whole-mount staining reveals that cecal colonization by vagal $\mathrm{NC}$ cells is well under way at E14.5 in the WT and $\mathrm{ET}_{\mathrm{B}}$ heterozygous rat but that only the very proximal colon contains NC-derived cells at this time point (Fig. 1A). In contrast, reduced staining was present in the distal small intestine of HSCR embryos compared with WT, and low-density staining was observed only as far distally as the cecum, even at E18.5 (Fig. 1B). Staining in the distal colon of 
A

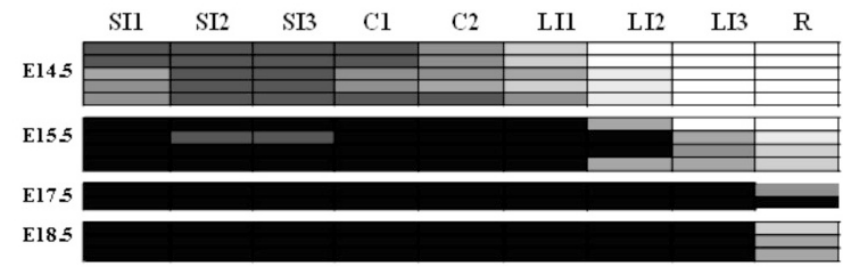

B.

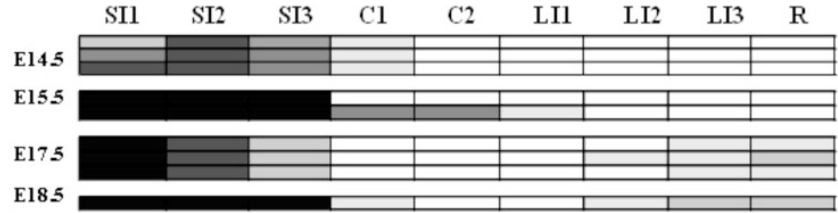

Figure 1. The pattern of NCSC colonization of the developing gut in WT and $\mathrm{ET}_{\mathrm{B}}$ heterozygous embryos. Whole-mount immunohistochemistry for $\mathrm{p} 75$ was performed on dissected embryonic guts, after which the guts were marked to three equal small intestinal (SI) sections, two cecal (C) sections, three equal large intestinal (LI) sections, and the rectum. Each section was scored on a six-point scale for the density and the intensity of staining in a genotype- and section-blinded manner. The results from $14 \mathrm{WT} / \mathrm{ET}_{\mathrm{B}}$ heterozygous (nine WT, five heterozygous) and nine HSCR intestines are schematically presented with the intensity of the box shading correlating with the p75 staining score (white is no staining, black is most intense staining). (A) $\mathrm{WT}^{-\mathrm{ET}_{\mathrm{B}}}$ heterozygous embryos. NC cells are progressing through the cecum and beginning to enter the large intestine at E14.5. NC cells have reached to rectum by E17.5. (B) HSCR embryos. NC cells have progressed caudally only to the proximal cecum at E14.5. At E15.5, small intestinal colonization seems to be complete, but colonization of the cecum remains delayed. At E17.5 and E18.5, staining is noted in the rectum and distal large intestine, where only large nerve trunks are identified.

HSCR rats was limited to large extrinsic nerve trunks in the distal colonic segments (Fig. 2).

Next we examined NCSC colonization of the developing rat intestine prospectively by isolation of $\mathrm{p} 75 / \alpha_{4}{ }^{\text {high }}$ cells. In WT embryos, we found a cranial-to-caudal progression in the number of $\mathrm{p} 75 / \alpha_{4}{ }^{\text {high }}$ cells that correlates well with our wholemount staining. At E13.5, there is a dramatic increase in the number of $\mathrm{p} 75 / \alpha_{4}{ }^{\text {high }}$ cells in the cecal segment. The number of $\mathrm{p} 75 / \alpha_{4}{ }^{\text {high }}$ cells begins to rise in the distal segment at E14.5 and reaches its peak at E16.5. It is interesting that in the proximal segment, the number of $\mathrm{p} 75 / \alpha_{4}{ }^{\text {high }}$ cells increases after E13.5, behind the $\mathrm{p} 75 / \alpha_{4}{ }^{\text {high }}$ colonization wavefront, at peaks at E15.5 (Fig. 3A).

Retrospective identification of NCSCs by culture of unselected, dissociated cells in the WT intestine gives a similar pattern. Although it does not reach statistical significance, the number of NCSCs trends upward in the proximal segment after E13.5. As with prospective sorting, after E13.5, there is a dramatic increase in the number of NCSCs in the cecal segment. This technique does not identify a dramatic increase in distal segment NCSCs until after E16.5 (Fig. 3B).

Culture of selected $\mathrm{p} 75 / \alpha_{4}{ }^{\text {high }}$ cells reveals that a high percentage $(50-80 \%)$ of the resultant colonies were multilineage - that is, they contained cells that stained for neural, glial, and smooth muscle markers. The proximal gut $\mathrm{p} 75 / \alpha_{4}$ high population not only increases in number from E13.5 to E15.5 but also becomes more likely to form multilineage colonies (Fig. 3C).
NCSCs do not colonize the cecum or distal gut of HSCR embryos. Prospective sorting with a gate set to collect the top $1 \%$ of cells that express p 75 and $\alpha_{4}$ in the WT proximal gut section demonstrates no change in the number of $\mathrm{p} 75 / \alpha_{4}$ high cells in the proximal gut of HSCR rats between E13.5 and E17.5 (Fig. 4A). A small number of $\mathrm{p} 75 / \alpha_{4}{ }^{\text {high }}$ cells are identified in the cecal and distal gut segments at these time points; however, culture of unselected dissociated gut cells fails to detect significant numbers of NCSC in these gut segments at any time point studied (Fig. 4B).

Culture of $\mathrm{p} 75 / \alpha_{4}{ }^{\text {high }}$ cells also fails to yield multilineage colonies (Fig. 4C). These cells were selected using a less restrictive gate (set to collect the top $1 \%$ of p75- and $\alpha_{4^{-}}$ expressing cells in the proximal section of the same embryo) to address the possibility that $\mathrm{ET}_{\mathrm{B}}$ deficiency affects the expression of p75 or $\alpha_{4}$

The NCSC pool in the WT but not the HSCR proximal gut increases behind the NC colonization wavefront. We compared the number of NCSCs in WT and HSCR embryos at different time points in the stomach and small intestine by prospective sorting and retrospectively by culture at clonal density of either dissociated, unselected intestinal cells or selected $\mathrm{p} 75 / \alpha_{4}{ }^{\text {high }}$ cells. The number of stomach and small intestinal cells that express high levels of p75 and $\alpha_{4}$ is reduced in HSCR embryos compared with WT after E14.5, with the most dramatic differences occurring at E14.5 and E15.5 (Fig.

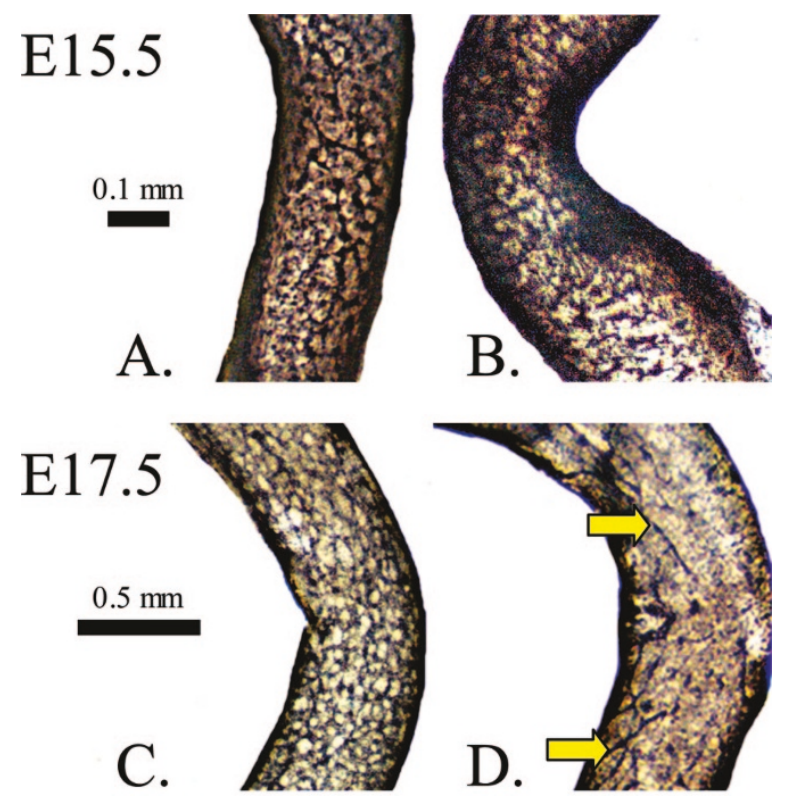

Figure 2. Whole-mount immunohistochemistry for p75 in WT and HSCR rats. Representative sections of the terminal ileum at E15.5 demonstrate an equal high-intensity p75 immunostaining between WT $(A)$ and HSCR $(B)$ embryos. No differences in staining pattern between the two genotypes are identified with this technique. At E17.5, representative sections demonstrate high-intensity p75 immunostaining in the distal colon of WT embryos $(C)$ and very low-intensity staining in the distal colon of HSCR embryos $(D)$. The staining pattern in the distal colon of HSCR embryos is markedly different from that observed in WT embryos. While WT embryos exhibit a fine, homogeneous meshwork pattern of staining, the HSCR embryos exhibit thick, irregularly spaced cords of staining (arrows in $D$ ), consistent with enlarged extrinsic nerve trunks. All sections are shown with the proximal end at the upper portion of the photograph. 

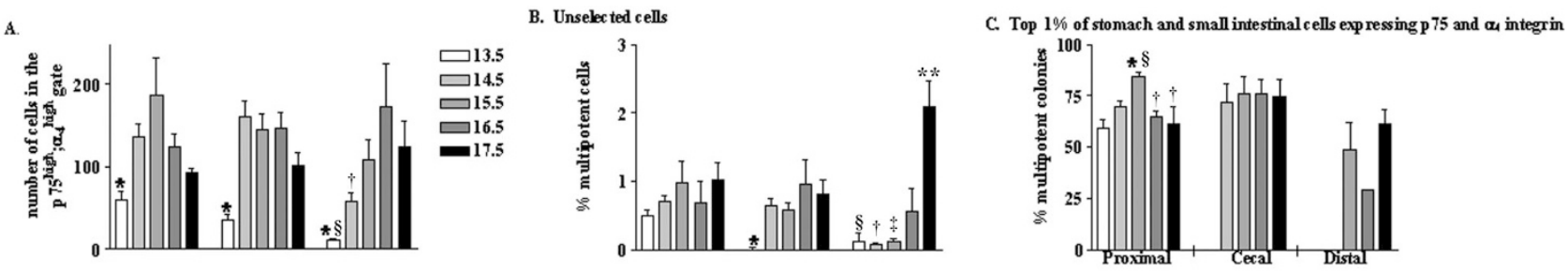

Figure 3. The pattern of NCSC colonization of the developing WT rat gut. Embryonic guts were dissected and divided into proximal (stomach to the ileocecal junction), cecal (cecum only), and distal (colon distal to cecum through rectum) segments. (A) Prospective sorting of cells with high levels of p75 and $\alpha_{4}$ expression. Gates were set to collect the top $1 \%$ of p $75 / \alpha_{4}$-expressing cells in the WT proximal section. A significant increase in the number of p $75 / \alpha_{4}{ }^{\text {high }}$ cells occurs in all segments between E13.5 and E14.5 with peak numbers of cells identified in the proximal and cecal sections at $\sim$ E15.5 at peak numbers of cells identified in the distal segment at E16.5. ${ }^{*} p \leq 0.05 v s$ later time points in the same segment; ${ }^{\S} p=0.001 v s$ proximal segment at same time point; ${ }^{\dagger} p=0.0002$ $v s$ cecal segment at same time point. $(B)$ Unselected cells from dissociated intestinal segments were cultured at clonal density, and the percentage of plated cells that formed colonies that contained neurons (Peripherin $\left.{ }^{\dagger}\right)$, glia $\left(\mathrm{GFAP}^{\dagger}\right)$, and myofibroblasts $\left(\mathrm{SMA}^{\dagger}\right)$ are indicated. $* p \leq 0.01 v s$ E13.5 proximal segment and E14.5, E15.5, and E17.5 cecal segment; ${ }^{\S} p<0.05 v s$ E13.5 proximal segment and E17.5 distal segment; ${ }^{\dagger} p<0.01 v s$ E14.5 proximal or cecal segments and E17.5 distal segment; ${ }^{\star} p<0.05$ vs E15.5 proximal or cecal segments and E17.5 distal segment; $* * p<0.04$ vs E17.5 proximal or cecal segments. (C) Cells that expressed high levels of p75 and $\alpha_{4}$ were cultured at clonal density, and the percentage of colonies that were multipotent are presented. The gates were set to isolate the top $1 \%$ of cells that expressed p75 and $\alpha_{4}$ in the proximal segment of each embryo. ${ }^{*} p<0.0004 v s$ E13.5 proximal bowel; ${ }^{\circledR} p<0.01 v s$ E14.5 proximal bowel; ${ }^{\dagger} p<0.05 v s$ E15.5 proximal bowel. The number of NC-derived colonies derived from the cecum at E13.5 and from the colon at E13.5 and E14.5 was too small $(<10)$ to reasonably calculate a percentage. All data $(A-C)$ are presented as the mean \pm SE, where $n=3-16$.

$5 A$ ). There was no significant difference between the two genotypes in the percentage of p75-positive cells in the p75/ $\alpha_{4}{ }^{\text {high }}$ gate. Similarly, the number of NCSCs in the proximal gut of WT rats is greater than that of the HSCR rat after E13.5 by unselected culture (Fig. 5B). Culture of selected cells does not reveal this difference until after E14.5 (Fig. 5C). This difference in the percentage of multipotent colonies does not seem to be related to a decreased ability of HSCR p $75 / \alpha_{4}{ }^{\text {high }}$ cells to survive and form colonies in culture because the difference was observed even in plates with high colony efficiencies, and colony efficiencies were not different between the two genotypes (data not shown).

Culture does not identify altered developmental pathway of cells that express high levels of p75 and $\alpha_{4}$ in the HSCR intestine. It is proposed that HSCR occurs in the absence of $\mathrm{ET}_{\mathrm{B}}$ signaling as a result of increased and premature neuronal differentiation of multipotent ENS precursors. If this is the case, then we might observe altered developmental outcomes in vitro. In addition, the top $1 \%$ of $\mathrm{p} 75 / \alpha_{4}{ }^{\text {high }}$ gate in the HSCR rat accepts lower levels of expression and, therefore, may select a slightly different population of cells. These cells may be capable of forming only more developmentally restricted colonies in culture. To examine whether the reduced number of multipotent NC-derived cells in the proximal gut of HSCR rats at E15.5 results from restriction of the developmental potential of the selected cells, we analyzed the composition of all colonies derived from the top $1 \% \mathrm{p} 75 / \alpha_{4}{ }^{\text {high }}$ cells in either genotype in culture. Despite finding reduced numbers of colonies forming all three cell types (neurons, glia, and myofibroblasts), we were unable to identify a difference in the number of colonies that formed only one or two of these cell types between WT and HSCR rats (data not shown). A total of $84 \pm 2 \%$ of WT p $75 / \alpha_{4}{ }^{\text {high }}$ cells formed trilineage colonies in culture, whereas only $54 \pm 2 \%$ of HSCR p $75 / \alpha_{4}{ }^{\text {high }}$ cells formed trilineage colonies in culture $(p=0.0005 ; n=6-8)$.
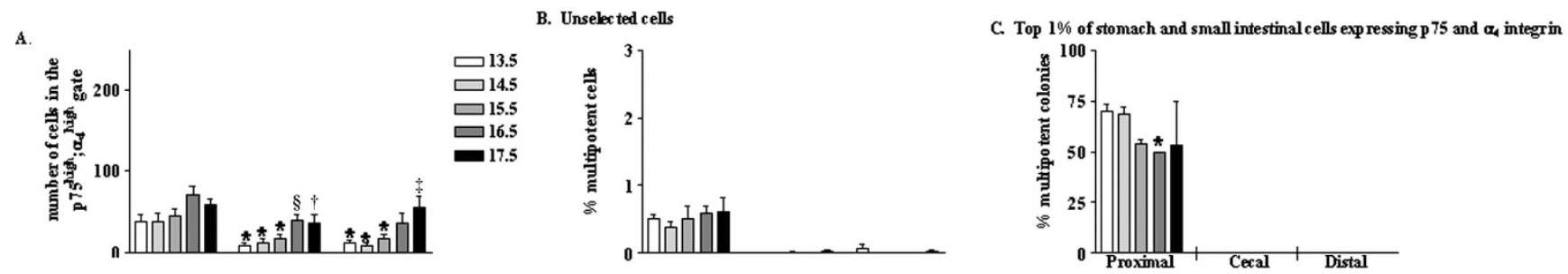

Figure 4. The pattern of NCSC colonization of the developing gut in HSCR embryos. Embryonic guts were dissected and divided into proximal (stomach to the ileocecal junction), cecal (cecum only), and distal (colon distal to cecum through rectum) segments. (A) Prospective sorting of cells with high levels of p75 and $\alpha_{4}$ expression. Gates were set to collect the top $1 \%$ of $\mathrm{p} 75 / \alpha_{4}$-expressing cells in the WT proximal section. There was no change in the number of $\mathrm{p} 75 / \alpha_{4}$ high cells in the proximal segment over the time points studied. Cecal and distal segments contained a reduced number of p $75 / \alpha_{4}{ }^{\text {high }}$ cells compared with the proximal segment at E13.5, E14.5, and E15.5. An increase in the number of $\mathrm{p} 75 / \alpha_{4}{ }^{\text {high }}$ cells is identified in cecal and distal segments beginning at E16.5. $* p \leq 0.05 v s$ the proximal segment at the same time point; ${ }^{\circledR} p<0.02$ vs E13.5, E14.5, and E15.5 cecal segments; ${ }^{\dagger} p \leq 0.05 v s$ E13.5 and E14.5 cecal segments; ${ }^{\ddagger} p<0.04$ $v s$ E13.5, E14.5, and E15.5 distal segments. (B) Unselected cells from dissociated intestinal segments were cultured at clonal density, and the percentage of plated cells that formed colonies that contained neurons (Peripherin $\left.{ }^{\dagger}\right)$, glia $\left(\mathrm{GFAP}^{\dagger}\right)$, and myofibroblasts $\left(\mathrm{SMA}^{\dagger}\right)$ are indicated. Multipotent NCSCs were virtually absent from the HSCR cecum and distal gut segments. (C) Cells that expressed high levels of p75 and $\alpha_{4}$ were cultured at clonal density, and the percentage of colonies that were multipotent are presented. The gates were set to isolate the top $1 \%$ of cells that expressed p75 and $\alpha_{4}$ in the proximal segment of each embryo. Despite collecting more cells than the WT proximal section gate (illustrated in $A$ ), no multipotent NC colonies are identified at any time point in the cecal and distal segments. ${ }^{*} p<0.03$ vs E13.5 proximal bowel. All data $(A-C)$ are presented as the mean \pm SE, where $n=3-6$. 
A

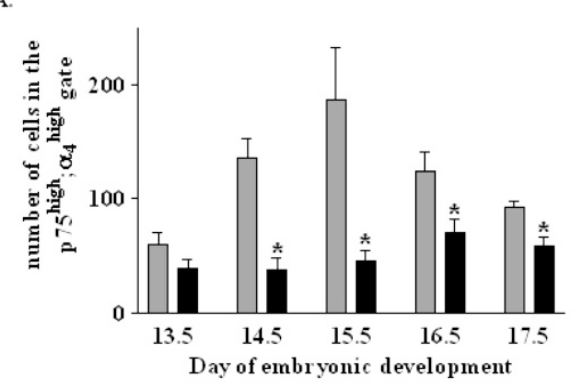

B

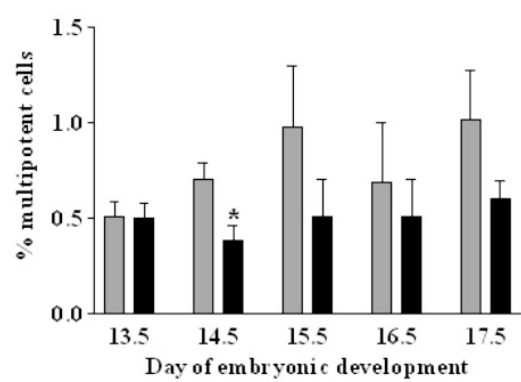

C.

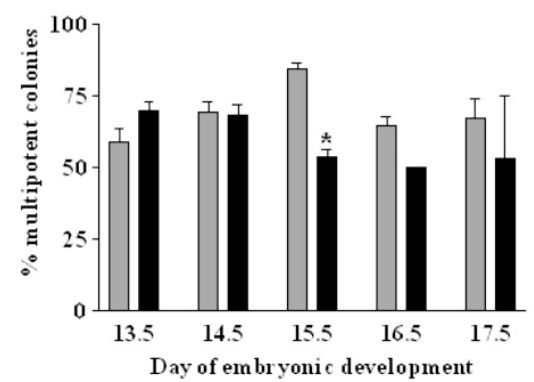

Figure 5. WT rats (畻) have more NCSCs that HSCR rats $(\square)$ in the small intestine after E14.5. (A) Dissociated small intestinal segments (stomach through ileocecal junction) were sorted for high p75 and $\alpha_{4}$ expression using a gate defined by the top $1 \%$ of cells that expressed these markers in the proximal segment of WT embryos of the same age, stained and sorted on the same day. There is a dramatic difference in the number of cells with this phenotype in the proximal gut of WT and HSCR embryos beginning at E14.5. ${ }^{*} p<0.05 v s \mathrm{WT}$ at the same time point; $p<0.0001$ for the affect of genotype and $p=0.01$ for the affect of time on the number of these cells by ANOVA with a significant interaction between the two variables. (B) Unselected, dissociated small intestinal segment cells were cultured at clonal density, and the percentage of cells that gave rise to multipotent NC-derived colonies (NCSCs) is presented. At E14.5, there were significantly more NCSCs in the in WT compared with HSCR embryos. * $p<0.03 v$ WT on the same day; $p=0.03$ for the affect of genotype on the percentage of multipotent cells in the proximal segment by ANOVA. (C) Cells that expressed high levels of p75 and $\alpha_{4}$ were selected from the proximal segment (stomach through ileocecal valve) using the gate defining the top $1 \%$ of cells that expressed these markers in WT embryos of the same age, stained and sorted on the same day. Cells were cultured at clonal density for 2 wk followed by immunostaining for expression of neuron $\left(\right.$ Peripherin $\left.^{+}\right)$, glial $\left(\mathrm{GFAP}^{+}\right)$, or myofibroblast $\left(\mathrm{SMA}^{+}\right)$markers. Despite that there is no difference in the percentage of sorted cells that gave rise to NC-derived colonies between the two groups (data not shown), there is a significant difference in the percentage of multipotent colonies between WT rats and HSCR rats. $* p=0.0005 v s$ WT at E15.5; $p=0.04$ for the affect of genotype of the percentage of multipotent colonies in the proximal gut section. To prevent wide swings in the percentage data, only cultures that gave rise to $\geq 10 \%$ NC-derived colonies are presented in $A$ and $B$. Data are presented as the mean \pm SE, where $n=4-16$.

Reduced numbers of NCSCs in the stomach and small intestine of HSCR rats is not associated with a generalized reduction in proliferation of these cells. We examined the cell-cycle distribution in the proximal segment of the developing gut from E13.5 to E17.5. Because we were interested in determining whether there is a generalized proliferation defect in HSCR NCSCs, we removed the last $20 \%$ of small intestine from this analysis so that we could compare regions outside the known colonization defect in $\mathrm{ET}_{\mathrm{B}}$ deficiency. In the stomach and proximal four fifths of the small intestine, we found no difference in the percentage of mitotically active cells using Hoechst 33342 staining of both unselected cells and $\mathrm{p} 75 / \alpha_{4}{ }^{\text {high }}$ cells between WT and HSCR rats (Fig. 6). We found a progressive decrease in proliferative activity of these cells between E13.5 and E17.5 in both genotypes.

\section{DISCUSSION}

The ability to prospectively identify NCSCs from the developing gut is a powerful research tool and is extremely important in the development of new therapeutic strategies to treat diseases of the ENS. In this study, we detected the maintenance of and regional increases in the NCSC pool behind the colonization wavefront in WT rat embryos. We also compared the dynamics of the NCSC pool between WT rats and HSCR rats and found a significant relative reduction in the HSCR stem cell pool in the small intestine after E13.5-a time when colonization of the rat small intestine seems to be complete.

The original report by Ikadai et al. (3) found that $59 \%$ of neonatal $\mathrm{ET}_{\mathrm{B}}$-deficient rats exhibited aganglionosis proximal to the ileocecal junction, and abnormalities in the small intestinal $\mathrm{ENS}$ in postnatal $\mathrm{ET}_{\mathrm{B}}$-deficient rats and $\mathrm{ET}_{3}$-deficient mice are reported $(21,22)$. Delayed embryologic development of the small intestinal ENS in HSCR rats is also described (23).
Recently, Barlow et al. (24) quantified undifferentiated NCderived cells in $\mathrm{ET}_{3}$-deficient mouse small intestinal sections using a Phox2B-specific riboprobe. They found an $\sim 43 \%$ reduction in cell number in HSCR sections compared with WT sections at E12.5 but not at E15.5. This is despite the fact that the colonization wavefront had reached the ileocecal junction by $\mathrm{E} 12.5$ in $\mathrm{ET}_{3}$-deficient mice and that these mice have normal numbers of myenteric neurons in the ileum postnatally (22). Consistent with our findings in the rat, they concluded that $\mathrm{ET}_{\mathrm{B}}$ signaling deficiency in the mouse leads to transient reductions in the number and delayed caudal progression of undifferentiated NC-derived cells (24).

Using a FACS gate that selects the top $1 \%$ of $\mathrm{p} 75 / \alpha_{4^{-}}$ expressing cells in the WT proximal section, we found a significant reduction in this population in the HSCR rat embryos in the small intestine after E13.5. When we allowed the gate to be defined by each embryo and selected the top $1 \%$ of cells that expressed p75 and $\alpha_{4}$, we found that the selected cells from HSCR stomach and small intestine gut sections were less capable of forming multipotent colonies after E14.5. We suspect that this results from the selection of cells with relatively lower levels of p75 and $\alpha_{4}$ expression-cells that prove in culture not to be multipotent. Taken together, the decreased number of $\mathrm{p} 75 / \alpha_{4}{ }^{\text {high }}$ cells and the reduction in multipotent colony formation in both selected and unselected cultures suggest that HSCR rats have a reduced NCSC pool in the small intestine compared with WT rats after colonization of this region of the gut.

It is interesting that the NCSC pool in the small intestine of WT rats significantly increases between E13.5 and E15.5, when the colonization wavefront is moving through the proximal colon. This raises the issue of the interaction between the number of undifferentiated NC-derived cells in the gut and 


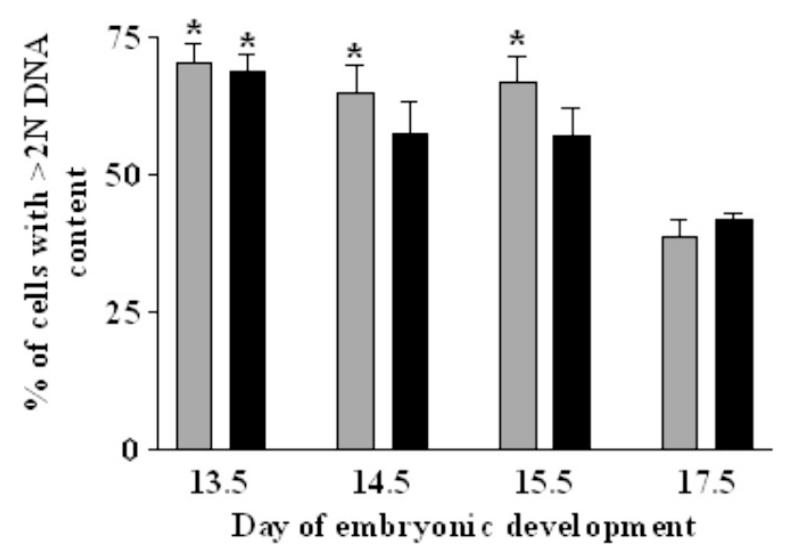

Figure 6. No difference in NCSC proliferation is detected in the proximal gut of WT compared with HSCR embryos. Intestinal segments were dissociated on the indicated embryonic day. The top $1 \%$ of cells that expressed p75 and $\alpha_{4}$ in the stomach and proximal four fifths of the small intestine were subjected to cell-cycle analysis using Hoechst 33342 staining and flow cytometry. No differences between WT (畻) and HSCR ( $\square$ ) embryos were detected. By ANOVA, mitotic activity significantly decreased with time $(p<0.0001)$. $* p<$ 0.005 by $t$ test $v s$ the percentage of cells of the same genotype with $>2 \mathrm{~N}$ DNA content at E17.5. Data are presented as mean $\pm \mathrm{SE} ; n=2-10$.

their ability to migrate. Several studies have shown that reduction in the number of vagal crest cells in vivo reduces the distance that these cells travel in the developing gut (13-15). Young et al. (13) found that if a small population of the most caudal NC-derived cells in the mouse colon is isolated from the population behind them, the cells migrate more slowly. NCderived cells that were not in contact with other NC-derived cells were immobile in the gut mesenchyme. These findings support the concept that migration may result from "population pressure" behind the leading edge of cells. The development of a large NCSC pool behind the colonization wavefront in WT rats may play a role in the successful colonization of the cecum and proximal colon.

In the mouse, the requirement for $\mathrm{ET}_{\mathrm{B}}$ activation is restricted to the time when NC-derived cells are colonizing the pericecal region $(25,26)$. This timing and localization are consistent with observations of pronounced abnormal NC cell behavior beginning in the distal small intestine of $\mathrm{ET}_{3}$-deficient mice (27) and with high levels of $\mathrm{ET}_{3}$ expression beginning during this time in the mesenchyme of the developing cecum (24). On the basis of extensive observation of ENS precursor colonization patterns in $\mathrm{ET}_{3}$-deficient and WT mice, Coventry et al. (27) hypothesized that expansion of the pool of undifferentiated NC cells may act as a "driving force" for colonization of the proximal colon. Defects in $\mathrm{NC}$ colonization of the $\mathrm{ET}_{3}$ deficient cecum therefore could result from a failure to expand the pool of undifferentiated cells in or proximal to this region.

Sidebotham et al. (28) and Young et al. (29) reported colonization of the entire hindgut in organ culture when segments of proximal gut that contained NC cells were apposed with postcecal colon that lacked NC cells. This may argue against an important role of an expanding NCSC population in the pericecal region for complete hindgut colonization, except that colonization of the hindgut in organ co-culture is significantly different from colonization in vivo. Sidebotham et al.
(28) reported that the proximal gut NC cells "demonstrated equivalent ability to migrate to and differentiate in the terminal rectum" as NC cells at the leading edge of colonization in the cecum. However, they noted that the pattern of colonization of the colon in the apposition experiments differed from that of whole gut in vivo and in vitro in that the number of cells was much reduced, the NC cells were largely restricted to the half of the colon adjacent to the site of apposition, and the NCderived cells predominately migrated superficially around the surface of the apposed terminal rectum with only small numbers of cells penetrating into the mesenchyme (28). Young et al. (29) also noted differences between in vivo colonization of the hindgut by NC cells and colonization in their co-culture experiments. They noted different patterns of marker expression in ENS progenitors in vitro compared with in vivo. They also noted that the growth of cultured gut is considerably less than in vivo. Thus, NC cells that fail to completely colonize the gut in vivo may be able to colonize the hindgut in co-culture because they have less distance to migrate and less gut to colonize than in vivo (29). How well co-culture NC migration experiments reflect the in vivo colonization process is unclear.

The reduction in NCSCs in HSCR rats relative to WT rats may be due to restriction in their developmental potential/ premature differentiation, impaired proliferation, or increased apoptosis. The similar percentage of p75-positive cells selected as $\mathrm{p} 75 / \alpha_{4}{ }^{\text {high }}$ between the genotypes suggests that the reduction in the number of NCSCs in the HSCR rat is associated with a reduced number of p75-positive cells. This could result from loss of p75 expression with differentiation or a failure in NC cell proliferation. Previous studies have shown unaltered development of NCSCs from the E14.5 whole gut of the HSCR rat compared with WT in culture (7). We obtained similar results when we limited the analysis to just the proximal intestine at E15.5. In vitro differentiation conditions are, of course, extremely different from those encountered by NCSCs in vivo and may not reflect events within the HSCR gut.

We observed a nonsignificant trend for HSCR p $75 / \alpha_{4}{ }^{\text {high }}$ cells to be less successful in forming colonies over 2 wk in vitro. This suggests a proliferation defect. Barlow et al. (24) examined proliferative activity in undifferentiated ENS progenitors (using SOX10 expression as a marker) from the isolated small bowel of $\mathrm{ET}_{3}$-null mice at E11.5-E12, roughly corresponding to E13.5-E14 in the rat, using short-term culture. They found a significant reduction in the number of proliferating ENS progenitors. However, Woodward et al. (9) examined in situ proliferation of NC-derived cells using bromodeoxyuridine incorporation and found no difference between WT and $\mathrm{ET}_{3}$-deficient mice at E11.5. Whereas the results of Barlow et al. may be an artifact of cell culture, Woodward et al. may have been unable to identify the very small percentage of embryonic gut cells that are undifferentiated ENS progenitors. Using flow cytometry, we detected no difference in in vivo mitotic activity in $\mathrm{p} 75 / \alpha_{4}{ }^{\text {high }}$ cells in the stomach and proximal $80 \%$ of small intestine. Our data are consistent with the reduction in NCSCs in HCSR stomach and small intestines, being unrelated to changes in proliferative activity of these cells or resulting from very regional (distal $20 \%$ of small intestine) differences in proliferation. 
We did not examine whether HSCR rat NCSCs undergo apoptosis at increased rates compared with WT rats. Others have looked at this issue and found no increase in apoptosis associated with aganglionosis as a result of deficient $\mathrm{ET}_{\mathrm{B}}$ signaling (9). However, these studies concentrated on the leading edge of colonization. Increased apoptosis well behind the colonization wavefront may have been missed.

Kruger et al. (7) (including ourselves) used prospective identification of $\mathrm{p} 75 / \alpha_{4}$ high cells to compare the number of NCSCs in the whole gut and identified a $60 \%$ reduction in the number of these cells in the HSCR rat compared with the WT rat at $\mathrm{E} 12.5$ and $\mathrm{E} 14.5$. We proposed that before $\mathrm{E} 12.5 \mathrm{ET}_{\mathrm{B}}$ has an effect on NCSC survival or self-renewal that persists but does not worsen with time. Looking prospectively at the isolated small intestine at E13.5, we also identify a reduction $(\sim 43 \%)$ in the number of $\mathrm{p} 75 / \alpha_{4}{ }^{\text {high }}$ cells. However, culture with retrospective identification of NCSCs suggests that there are equal numbers of NCSCs in the two genotypes at this time point. In addition, in the current work, prospective sorting identified a population of cells in the HSCR cecum and distal colon that were incapable of forming multipotent colonies in culture. Not all cells that are selected on the basis of high p75 and $\alpha_{4}$ expression are NCSCs. Figure 5C illustrates that 20$50 \%$ of the NC-derived colonies formed from these cells are not multipotent. Another possible explanation for the discrepancy between prospectively and retrospectively identified NCSCs in the HSCR rat colon is that our hindgut dissections included sacral crest cells that populate pelvic ganglia in the mesenchyme surrounding the hindgut and are not found in the gut before the arrival of vagal crest cells (at least in the mouse) (30). Because we also found that these cells were incapable of forming multipotent colonies, we would have to speculate that the sorted cells are either not multipotent or that NCSCs that are isolated from this region respond differently to our culture conditions. Bixby et al. (6) found that that NCSCs that were isolated from the rat gut at E14.5 (which would be largely derived from the vagal crest) were more responsive to neurogenic factors than NCSCs that were isolated from the sciatic nerve. Clearly, caution must be used in interpreting the results of prospective identification and quantification of NCSCs without supporting culture data.

The ability to define phenotypically and isolate prospectively the population of enteric NCSCs from postnatal and adult mammals $(5,20)$ suggests the possibility of alternative therapeutic approaches to disorders of the ENS. Several groups have successfully injected undifferentiated NC cells into the fetal gut wall and observed neuronal development in organ culture $(7,31)$. Of course, neuronal and glial development is useless in the absence of functional connections. These connections start early as undifferentiated NC-derived cells migrate in chains along a scaffold created by the processes of leading edge cells (13). Therapeutic creation of a wellfunctioning ENS may require the induction of embryonic patterns of behavior in a transplanted or persistent NCSC population. Understanding the region-specific patterns of enteric NCSC behavior during normal colonization will aid the development of these new therapeutic approaches.
Acknowledgments. We thank Sean Morrison and Genevieve Kruger for help with the isolation and culture of NCSCs, Martin White and David Adams in the University of Michigan Flow Cytometry Core, and Richard Ransom for help in the University of Michigan Department of Pediatrics Imaging Facility.

\section{REFERENCES}

1. Imseis E, Gariepy CE 2004 Hirschsprung Disease. In: Walker WA (ed) Pediatric Gastrointestinal Disease: Pathophysiology, Diagnosis, and Management. BC Decker Inc., Hamilton, pp 1032-1043

2. McCallion AS, Chakravarti A 2004 RET and Hirschsprung disease and multiple endocrine neoplasia type 2. In: Epstein CJ, Erickson RP, Wynshaw-Boris A (eds) Inborn Errors of Development: The Molecular Basis of Clinical Disorders or Morphogenesis. Oxford University Press, New York, pp 421-432

3. Ikadai H, Fujita H, Agematsu Y, Imanichi T 1979 Observation of congenital aganglionosis rat (Hirschsprung's disease) and its genetical analysis (in Japanese). Cong Anom 19:31-36

4. Gariepy CE, Cass DT, Yanagisawa M 1996 Null mutation of endothelin receptor type $\mathrm{B}$ gene in spotting lethal rats causes aganglionic megacolon and white coat color. Proc Natl Acad Sci USA 93:867-872

5. Bondurand N, Natarajan D, Thapar N, Atkins C, Pachnis V 2003 Neuron and glia generating progenitors of the mammalian enteric nervous system isolated from foetal and postnatal gut culture. Development 130:6387-6400

6. Bixby S, Kruger GM, Mosher JT, Joseph NM, Morrison SJ 2002 Cell-intrinsic differences between stem cells from different regions of the peripheral nervous system regulate the generation of neural diversity. Neuron 35:643-656

7. Kruger GM, Mosher JT, Tsai YH, Yeager KJ, Iwashita T, Gariepy CE, Morrison SJ 2003 Temporally distinct requirements for endothelin receptor B in the generation and migration of gut neural crest stem cells. Neuron 40:917-929

8. Iwashita T, Kruger GM, Pardal R, Kiel MJ, Morrison SJ 2003 Hirschsprung disease is linked to defects in neural crest stem cell function. Science 301:972-976

9. Woodward MN, Sidebotham EL, Connell MG, Kenny SE, Vaillant CR, Lloyd DA, Edgar DH 2003 Analysis of the effects of endothelin-3 on the development of neural crest cells in the embryonic mouse gut. J Pediatr Surg 38:1322-1328

10. von Boyen GB, Krammer HJ, Suss A, Dembowski C, Ehrenreich H, Wedel T 2002 Abnormalities of the enteric nervous system in heterozygous endothelin B receptor deficient (spotting lethal) rats resembling intestinal neuronal dysplasia. Gut 51:414-419

11. Hearn CJ, Murphy M, Newgreen D 1998 GDNF and ET-3 differentially modulate the numbers of avian enteric neural crest cells and enteric neurons in vitro. Dev Biol 197:93-105

12. Wu JJ, Chen JX, Rothman TP, Gershon MD 1999 Inhibition of in vitro enteric neuronal development by endothelin-3: mediation by endothelin B receptors. Development 126:1161-1173

13. Young HM, Bergner AJ, Anderson RB, Enomoto H, Milbrandt J, Newgreen DF, Whitington PM 2004 Dynamics of neural crest-derived cell migration in the embryonic mouse gut. Dev Biol 270:455-473

14. Burns AJ, Champeval D, Le Douarin NM 2000 Sacral neural crest cells colonize aganglionic hindgut in vivo but fail to compensate for lack of enteric ganglia. Dev Biol 219:30-43

15. Peters-van der Sanden MJ, Kirby ML, Gittenberger-de Groot A, Tibboel D, Mulder MP, Meijers C 1993 Ablation of various regions within the avian vagal neural crest has differential effects on ganglion formation in the fore-, mid- and hindgut. Dev Dyn 196:183-194

16. Gariepy CE, Williams SC, Richardson JA, Hammer RE, Yanagisawa M 1998 Transgenic expression of the endothelin-B receptor prevents congenital intestinal aganglionosis in a rat model of Hirschsprung disease. J Clin Invest 102:1092-1101

17. Stemple DL, Anderson DJ 1992 Isolation of a stem cell for neurons and glia from the mammalian neural crest. Cell 71:973-985

18. Morrison SJ, Csete M, Groves AK, Melega W, Wold B, Anderson DJ 2000 Culture in reduced levels of oxygen promotes clonogenic sympathoadrenal differentiation by isolated neural crest stem cells. J Neurosci 20:7370-7376

19. Morrison SJ, Weissman IL 1994 The long-term repopulating subset of hematopoietic stem cells is deterministic and isolatable by phenotype. Immunity 1:661-673

20. Kruger GM, Mosher JT, Bixby S, Joseph NM, Iwashita T, Morrison SJ 2002 Neural crest stem cells persist in the adult gut but undergo changes in self-renewal, neuronal subtype potential, and factor responsiveness. Neuron 35:657-669

21. Nagahama M, Ozaki T, Hama K 1985 A study of the myenteric plexus of the congenital aganglionosis rat (spotting lethal). Anat Embryol (Berl) 171:285-296

22. Sandgren K, Larsson LT, Ekblad E 2002 Widespread changes in neurotransmitter expression and number of enteric neurons and interstitial cells of Cajal in lethal spotted mice: an explanation for persisting dysmotility after operation for Hirschsprung's disease? Dig Dis Sci 47:1049-1064

23. Newgreen DF, Hartley L 1995 Extracellular matrix and adhesive molecules in the early development of the gut and its innervation in normal and spotting lethal rat embryos. Acta Anat (Basel) 154:243-260

24. Barlow A, de Graaff E, Pachnis V 2003 Enteric nervous system progenitors are coordinately controlled by the G protein-coupled receptor EDNRB and the receptor tyrosine kinase RET. Neuron 40:905-916

25. Shin MK, Levorse JM, Ingram RS, Tilghman SM 1999 The temporal requirement for endothelin receptor-B signaling during neural crest development. Nature 402:496501 
26. Woodward MN, Kenny SE, Vaillant C, Lloyd DA, Edgar DH 2000 Time-dependent effects of endothelin-3 on enteric nervous system development in an organ culture model of Hirschsprung's disease. J Pediatr Surg 35:25-29

27. Coventry S, Yost C, Palmiter RD, Kapur RP 1994 Migration of ganglion cell precursors in the ileoceca of normal and lethal spotted embryos, a murine model for Hirschsprung disease. Lab Invest 71:82-93

28. Sidebotham EL, Woodward MN, Kenny SE, Lloyd DA, Vaillant CR, Edgar DH 2002 Localization and endothelin-3 dependence of stem cells of the enteric nervous system in the embryonic colon. J Pediatr Surg 37:145-150
29. Young HM, Jones BR, McKeown SJ 2002 The projections of early enteric neurons are influenced by the direction of neural crest cell migration. J Neurosci 22:60056018

30. Kapur RP 2000 Colonization of the murine hindgut by sacral crest-derived neural precursors: experimental support for an evolutionarily conserved model. Dev Biol 227:146-155

31. Natarajan D, Grigoriou M, Marcos-Gutierrez CV, Atkins C, Pachnis V 1999 Multipotential progenitors of the mammalian enteric nervous system capable of colonising aganglionic bowel in organ culture. Development 126:157-168 